\title{
Functional Status of Women and Their Partners after Childbirth
}

\author{
Sevgi Özkan (iD) 1, ${ }^{*}$, Sinem Göral Türkcü (D)1, Özlem Kayhan', \\ Pınar Serçekuş Ak (iD), Ümran Sevil²
}
${ }^{1}$ Pamukkale University, Faculty of Health Sciences, Department of Obstetrics and Gynecology Nursing, Denizli, Turkey
${ }^{2}$ Ege University, Nursing Faculty, Department of Obstetrics and Gynecology Nursing, İzmir, Turkey

\begin{abstract}
The aim of this study is to define the changes within the social dimension of the functional status of women and their partners in the months following the birth of their child. A total of 90 participants were included in the study (45 women and 45 partners). Data were collected in two steps via face-to-face interviews. The first step involved administration of a personal information form and the functional status inventories to the women and their partners between the postpartum sixth and eighth weeks. The second step involved re-administration of the same functional status inventories to the women and their partners in the postpartum sixth month. Results from the examination of the functional status of the women and their partners in the postpartum second and sixth months showed that there was a significant increase in the areas of household activities $(\mathrm{p}=0,000)$, social and community activities $(p=0,000)$ and self-care activities $(p=0,000)$ for the women in the sixth month; similarly, for the fathers, household activities $(\mathrm{p}=0,008)$, social and community activities $(\mathrm{p}=0,003)$ and child care activities $(\mathrm{p}=0,007)$ rose in the sixth month. Furthermore, the study found that certain sociodemographic variables had an effect on functional status $(p=0,000)$. It is important that nurses and/or midwives support parents during the pregnancy process, particularly during the transition into their new roles as women and their partners, and help prepare them for the changes to occur as they enter into this new period of life.
\end{abstract}

\section{ARTICLE HISTORY}

Received: 27 April 2018

Revised: 19 June 2018

Accepted: 12 July 2018

\section{KEYWORDS}

Functional Status, Transition to Motherhood, Transition to Fatherhood

\section{INTRODUCTION}

In the postpartum period, much of the focus in the studies conducted has tended to center on recovery of the reproductive organs. On the other hand, some topics such as the process of transition to parenthood, increased responsibilities, tiredness, changes in the relationship with the spouses, pre-pregnancy social and professional activities are not considered enough (Fichardt, Van Wyk \& Weich, 1994; Gjerdingenm \& Chaloner, 1994; Herbert, 1998; Hodnett, 1996). The functional status of women are required to "take on the responsibility of infant care,

CONTACT: Sevgi Özkan $\varangle$ sozkan@pau.edu.tr Ð Pamukkale University, Faculty of Health Sciences, Campus Kınıklı, 20070, Denizli/ Turkey. 
self-care, household activities, and social, community, and occupational activities" (Fawcett, Tulman \& Myers, 1988). The functional status of fathers, on the other hand, either stays the same or expands to involve more responsibilities, including those related to social, community, occupational, and educational activities, and child and personal care. Furthermore, partners take on the responsibility of taking care of other children within the family during pregnancy and after the childbirth (McVeigh, 2001; Tulman, Fawcett \& Weiss, 1993).

In some studies, parents have described the postpartum period as a process that generates major problems for both themselves and their family life. Moreover, research has shown that it requires women a longer amount of time to return to their antenatal functional status than to recover physiologically (Fawcett et al., 1988; Fichardt et al., 1994). Identifying precisely how the functional status of women changes in the postpartum period, as well as the factors that are brought to bear on these changes, is therefore key. During the postpartum period in particular, which is marked by an increasing number of difficulties, it is necessary to focus on solutions, not only to the physical problems women experience, but also to their social and psychological conditions, as these tend to take a longer time to overcome (Apay, Ejder \& Pasinlioğlu, 2009; Beji, Coşkun \& Yıldırım, 2003; Özkan \& Sevil, 2007).

The men's transition to fatherhood is as complicated as the woman's transition to motherhood. The role of fatherhood has been shown to start at the diagnosis of pregnancy, continuing up to the birth of the baby and evolving during the months following the birth (Barclay \& Lupon, 1999; Başbakkal, 1999; Sevil \& Özkan, 2010). This period requires that the father takes on more responsibility, remains committed, and allocates sufficient time for the infant. In this period of transition, men experience a number of changes in their lifestyle, including having to face the facts about pregnancy and child birth, making efforts to accept fatherhood, and adapting to a new role (Anderson, 1996). "Expectant" fathers may experience anxiety, conflicting emotions, weakness and happiness, all of which enable them to develop a new set of skills necessary for the roles related to fatherhood (Barclay, Donovan, \& Genovese, 1996; Henderson \& Brouse, 1991). The functional status of fathers is a multidimensional process, and the transition to motherhood and fatherhood can be a complicated period for both women and their partners. In this process, men are expected to not only fulfil their previous duties but also to get accustomed to their new and challenging role of being father (Fawcett et al., 1988; McVeigh, 2000a; McVeigh, 2000b; Sevil \& Özkan, 2010; Tulman et al., 1993).

Most studies have largely concentrated on the womens' functional status before and after the birth. There is not enough information about the functional status of both women and their partners in the postpartum period (Apay et al., 2009; Beji et al., 2003; Fichardt et al., 1994; McVeigh \& Chaboyer, 2002; Özkan \& Sevil, 2007; Posmontier, 2008; Şanlı \& Öncel, 2014; Tulman, Fawcett, Groblewski \& Silverman, 1990). For this reason, this study has aimed to determine the changes that occur within the social sphere, in terms of the postpartum functional status and recoveries of women and their partners, in the months following the birth, and also to determine the correlation between socio-demographic factors and postpartum functional status.

\section{MATERIALS AND METHODS}

\subsection{Data and test subject selection}

This descriptive study was conducted in order to identify the postpartum functional status of women and their partners. This study was conducted in three Family Health Centers which were low, moderate, and high socio-economic standings. The Family Health Centers were selected from 51 Family Health Centers located in the city center of Denizli, Turkey. The study involved women and their partners in the postpartum sixth to eighth weeks. Using a sampling size calculation formula, applicable for when the test subject is known, the number of 
participants was calculated to be 41 , at a confidence interval of $95 \%$ and a sampling error of $5 \%$; however, to preempt possible loss of participants, the study started out with 58 women and their partners. The study ended up being completed with 45 women and 45 partners (in total 90 participants).

\subsection{Inclusion criteria}

To participate in the study, the women had to have had a full-term vaginal delivery or caesarean section and be in the postpartum sixth to eighth weeks, while both mother and father had to have no mental retardation, chronic illness or disability, be able to speak and understand Turkish, and agree to participate in the study. All the infants in the study were full term without any health problems.

\subsection{Data collection tool}

The tools used in the study included a personal information form, which was developed based on corresponding literature, to determine the socio-demographic characteristics of the test subject; the Inventory of Functional Status After Childbirth (IFSAC), to evaluate the postpartum functional status of women; and the Inventory of Functional Status-Fathers (IFSF), to evaluate the functional status of fathers. Data collection involved a two-step process, which was conducted through face-to-face interviews. The first step included administration of the personal information form and the functional status inventories (IFSAC, IFS-F) to the women and their partners in the postpartum sixth to eighth weeks. The second step included re-administration of the same functional status inventories to the women and their partners in the postpartum sixth month.

\subsubsection{Personal information form}

Prepared on the basis of the corresponding literature, the questionnaire that ten questions included features questions about the socio-demographic characteristics and income status of participants, as well as the number of pregnancies and number of living children, delivery method, method of feeding their children, occupation, and the state of the support they give in infant care and household activities (Beji et al., 2003; Fawcett et al., 1988; McVeigh, 2000a; McVeigh, 2000b; Özkan \& Sevil, 2007).

\subsubsection{IFSAC: Inventory of functional status after childbirth}

The Inventory of Functional Status After Childbirth (IFSAC), which is based on the role function model of the Roy Adaptation Model, is used to measure functional status (Fawcett et al., 1988; Fawcett \& Tulman, 1990). The IFSAC questionnaire allows for the assessment of primary, secondary and tertiary roles in the postpartum period. There are five dimensional subscales of the inventory: infant care, self-care, household activities, social, community activities, and occupational activities. The validity and reliability were conducted by Özkan and Sevil (2007) in Turkey. The questionnaire consists of 36 items. Higher mean scores indicate greater functional status.

\subsubsection{IFS-F: The Inventory of functional status-fathers}

Developed to evaluate the functional status of fathers during the last trimester of pregnancy and the postpartum period (Tulman et al., 1993), this inventory was created on the basis of the role function model of the Roy Adaptation Model and features seven subscales: occupational activities, household activities, personal care activities, social and community activities, child care responsibilities, educational activities and baby care activities The validity and reliability were conducted by Sevil and Özkan (2010) in Turkey. The questionnaire consists of 51 items. Higher scores indicate a high functional status (McVeigh, 2001; Sevil \& Özkan, 2010; Tulman et al., 1993). 


\subsection{Ethical aspect of the study}

In order to conduct the study, permission was obtained from Denizli Provincial Directorate of Health and Non-Invasive Researches Ethics Committee of Pamukkale University. Also permission was obtained from the institution where the research was conducted. The women and their partners to be included in the study were informed about the purpose of the study and verbal and written consents were received from those who agreed to participate in the study.

\subsection{Data Analysis}

The data were analyzed using number values, percentage, mean, paired t-test, MannWhitney U Test, Wilcoxon Signed Ranks Test, and Kruskal Wallis analyses. Statistical significance was defined as $\mathrm{p}<0.05$.

\section{FINDINGS}

Table 1 shows the socio-demographic data of the women and their partners who participated in the study. In terms of the functional status of the women, a significant increase was found in the mean scores of household activities $(p=0.000)$, social and community activities $(\mathrm{p}=0.000)$ and self-care activities $(\mathrm{p}=0.000)$ in the postpartum sixth month. No significant difference, however, was determined between the months studied in terms of the mean scores obtained in the areas of infant care activities and occupational activities $(p>0.05)$ (Table 2).

Table 1. Socio-demographic characteristics of women and their partners

\begin{tabular}{lcc}
\hline Socio-Demographic Characteristics & Mother & Father \\
\cline { 2 - 3 } & $n(\%)$ & $n(\%)$ \\
\hline Residential area & & \\
Low & $15(33.3)$ & $15(33.3)$ \\
Moderate & $15(33.3)$ & $15(33.3)$ \\
High & $15(33.3)$ & $15(33.3)$ \\
Age & & \\
18-23 years & $4(8.9)$ & $0(0)$ \\
24-29 years & $17(37.8)$ & $9(20.0)$ \\
30-35 years & $16(35.6)$ & $21(46.7)$ \\
35 years and above & $8(17.7)$ & $15(33.3)$ \\
Educational Background & & \\
Illiterate & $1(2.2)$ & $0(0)$ \\
Primary school graduate & $12(26.7)$ & $14(31.1)$ \\
Secondary school graduate & $4(8.9)$ & $5(11.1)$ \\
High school graduate & $10(22.2)$ & $9(20.0)$ \\
Associate degree//Bachelor's degree & $18(40.0)$ & $17(37.8)$ \\
Occupation & & \\
Unemployed & $24(53.3)$ & $1(2.2)$ \\
Civil servant & $6(13.3)$ & $4(8.9)$ \\
Worker & $5(11.1)$ & $17(37.8)$ \\
Self-employed & $4(8.9)$ & $15(33.3)$ \\
Other & $6(13.4)$ & $8(17.8)$ \\
Economic condition & & \\
Income higher than expense & & \\
Income equal to expense & & \\
Income lower than expense & $11(24.4)$ & $10(22.2)$ \\
\hline & $26(57.8)$ & $22(48.9)$ \\
& $8(17.8)$ & $13(28.9)$ \\
\hline
\end{tabular}




\begin{tabular}{|c|c|c|}
\hline \multicolumn{3}{|l|}{$\overline{\text { Social security }}$} \\
\hline Yes & $43(95.6)$ & $42(93.3)$ \\
\hline No & $2(4.4)$ & $3(6.7)$ \\
\hline \multicolumn{3}{|l|}{ State of intending pregnancy } \\
\hline Intended and planned & $36(80.0)$ & $36(80.0)$ \\
\hline Intended but unplanned & $9(20.0)$ & $9(20.0)$ \\
\hline Common characteristics of Mother-Father & $\underline{\mathrm{n}}(\%)$ & \\
\hline \multicolumn{3}{|l|}{ Method of feeding the infant } \\
\hline Breast milk & $35(77.8)$ & \\
\hline Formula & $1(2.2)$ & \\
\hline Breast milk and formula & $9(20.0)$ & \\
\hline \multicolumn{3}{|l|}{ Contribution to infant care from the outside } \\
\hline Yes & $25(55.6)$ & \\
\hline No & $20(44.4)$ & \\
\hline \multicolumn{3}{|l|}{ Contribution to infant care $(n=25)$} \\
\hline Maternal/Grandmother-Paternal Grandmother & $16(35.6)$ & \\
\hline Father & $6(13.3)$ & \\
\hline Nanny & $3(6.7)$ & \\
\hline \multicolumn{3}{|l|}{ Contribution to household chores from the outside } \\
\hline Yes & $24(53.3)$ & \\
\hline No & $21(46.7)$ & \\
\hline \multicolumn{3}{|l|}{ Contribution to household chores $(n=24)$} \\
\hline Maternal Grandmother/Paternal Grandmother & $12(26.7)$ & \\
\hline Father & $9(20.0)$ & \\
\hline Nanny & $3(6.7)$ & \\
\hline \multicolumn{3}{|l|}{ Duration of marriage } \\
\hline $1-3$ years & $17(37.7)$ & \\
\hline 4-6 years & $11(24.4)$ & \\
\hline $7-10$ years & $10(22.2)$ & \\
\hline 10 years and more & $7(15.7)$ & \\
\hline \multicolumn{3}{|l|}{ Delivery method } \\
\hline Normal birth & $11(24.4)$ & \\
\hline Caesarean section & $34(75.6)$ & \\
\hline \multicolumn{3}{|l|}{ Number of pregnancies } \\
\hline 1 & $16(35.6)$ & \\
\hline 2 and more & $29(64.4)$ & \\
\hline \multicolumn{3}{|l|}{ Live birth } \\
\hline 1 & $17(37.8)$ & \\
\hline 2 and more & $28(62.2)$ & \\
\hline
\end{tabular}

Table 2. Functional status mean scores of women according to months

\begin{tabular}{lccc}
\hline Subscales & 2nd month $(n=45)$ & 6th month $(n=45)$ & \\
\cline { 2 - 3 } & Mean $\pm S D$ & Mean $\pm S D$ & \\
\hline Household activities & $2.59 \pm 0.49$ & $3.02 \pm 0.44$ & $\mathbf{. 0 0 0}^{*}$ \\
Social and community activities & $1.81 \pm 0.39$ & $2.13 \pm 0.51$ & $\mathbf{. 0 0 0}^{*}$ \\
Infant care activities & $3.75 \pm 0.26$ & $3.83 \pm 0.24$ & $.113^{*}$ \\
Self-care activities & $2.70 \pm 0.43$ & $3.08 \pm 0.28$ & $\mathbf{. 0 0 0}^{*}$ \\
Occupational activities $(n=2)$ & $2.40 \pm 0.00$ & $2.86 \pm 0.43$ & $.180^{* *}$ \\
\hline
\end{tabular}

\footnotetext{
*Paired t Test $\quad * *$ Wilcoxon Signed Ranks Test
} 
The examination of the fathers' functional status in the postpartum second and sixth months showed there to be a significant increase in the mean scores of household activities $(\mathrm{p}=0.008)$, social and community activities $(\mathrm{p}=0.003)$ and child care activities $(\mathrm{p}=0.007)$ in the sixth month, while no significant difference was found in terms of the months studied in the mean scores of infant care, self-care, and occupational and educational activities ( $p>0.05)$ (Table 3).

The effect of socio-demographic variables on the functional status of fathers was studied and analyzed as well. Fathers with a higher educational level had statistically significant mean scores in the area of infant care responsibilities in the postpartum eighth week $(p=0.024)$, and in the area of child care activities in the postpartum eighth week and the postpartum sixth month $(\mathrm{p}=0.023 ; \mathrm{p}=0.030)$. No significant difference was detected between the other subscales and educational background $(\mathrm{p}>0.05)$. Likewise, no significant difference was found between age, economic condition, number of children and the functional status in the postpartum second and sixth months ( $\mathrm{p}>0.05)$ (Table 4).

In examining the effect of socio-demographic variables on the functional status of women, it was discovered that women with a higher educational level had higher mean scores in the area of household activities in the postpartum sixth month $(\mathrm{p}=0.043)$. Furthermore, women who had a caesarean section were found to have stable mean scores in the area of household activities in the postpartum eighth week and higher mean scores in the postpartum sixth month, as compared to women's who gave normal birth (0.042). The other sociodemographic variables showed no significant influence on the women' functional status $(\mathrm{p}<0.05)$ (Table 5a, 5b).

Table 3. Functional status mean scores of partners according to months

\begin{tabular}{lccc}
\hline Subscales & 2nd month $(n=45)$ & 6th month $(n=45)$ & \multirow{2}{*}{$p$} \\
\cline { 2 - 3 } & Mean $\pm S D$ & Mean $\pm S D$ & \\
\hline Household activities & $2.34 \pm 0.94$ & $2.75 \pm 0.74$ & $\mathbf{. 0 0 8}^{*}$ \\
Social and community activities & $2.08 \pm 0.66$ & $2.47 \pm 0.61$ & $\mathbf{. 0 0 3}^{*}$ \\
Infant care activities & $2.24 \pm 0.96$ & $2.51 \pm 0.73$ & $.055^{*}$ \\
Child care activities $(n=29)$ & $2.51 \pm 0.84$ & $2.80 \pm 0.72$ & $\mathbf{. 0 0 7} *$ \\
Self-care activities & $2.73 \pm 0.53$ & $2.60 \pm 0.38$ & $.200^{*}$ \\
Occupational activities & $3.00 \pm 0.40$ & $3.03 \pm 0.20$ & $.697^{*}$ \\
Educational activities $(n=4)$ & $2.31 \pm 0.81$ & $3.00 \pm 0.00$ & $.180^{* *}$ \\
\hline
\end{tabular}

*Paired t Test **Wilcoxon Signed Ranks Test 
Table 4. The effect of some socio-demographic variables on the functional status of fathers

\begin{tabular}{|c|c|c|c|c|c|c|c|c|c|c|c|c|c|}
\hline \multirow[t]{2}{*}{ Variables } & \multicolumn{2}{|c|}{ Household responsibilities } & \multicolumn{2}{|c|}{$\begin{array}{l}\text { Social and community } \\
\text { activities }\end{array}$} & \multicolumn{2}{|c|}{$\begin{array}{l}\text { Infant care } \\
\text { responsibilities }\end{array}$} & \multicolumn{2}{|c|}{ Personal care activities } & \multicolumn{2}{|c|}{ Occupational activities } & \multicolumn{2}{|c|}{ Child care activities } & \multirow{2}{*}{$\begin{array}{l}\begin{array}{l}\text { Educational } \\
\text { activities }\end{array} \\
\text { 2nd month }\end{array}$} \\
\hline & 2nd month & 6th month & 2nd month & 6th month & 2nd month & 6th month & 2nd month & 6th month & 2nd month & 6th month & 2nd month & 6th month & \\
\hline 29 year and younger (n:9) & $2.26 \pm 0.78$ & $2.86 \pm 0.75$ & $1.95 \pm 0.68$ & $2.31 \pm 0.74$ & $2.37 \pm 1.08$ & $2.44 \pm 0.73$ & $2.74 \pm 0.67$ & $2.61 \pm 0.47$ & $3.00 \pm 0.50$ & $3.00 \pm 0.10$ & $1.95 \pm 0.06$ & $2.40 \pm 1.09$ & \\
\hline 30 year and over (n:36) & $2.36 \pm 0.98$ & $2.72 \pm 0.74$ & $2.12 \pm 0.66$ & $2.51 \pm 0.58$ & $2.21 \pm 094$ & $2.53 \pm 0.74$ & $2.72 \pm 0.50$ & $2.60 \pm 0.37$ & $3.00 \pm 0.38$ & $3.03 \pm 0.22$ & $2.55 \pm 0.86$ & $2.83 \pm 0.71$ & \\
\hline$p$ value & 0.875 & 0.809 & 0.305 & 0.359 & 0.694 & 0.875 & 0.919 & 0.919 & 0.546 & 0.755 & 0.227 & 0.630 & \\
\hline \multicolumn{14}{|l|}{ Education* } \\
\hline$\overline{\text { Secondary school and below (n:19) }}$ & $2.20 \pm 0.92$ & $2.59 \pm 0.76$ & $1.96 \pm 0.53$ & $2.32 \pm 0.64$ & $1.87 \pm 0.97$ & $2.34 \pm 0.77$ & $2.78 \pm 0.45$ & $2.51 \pm 0.43$ & $3.03 \pm 0.34$ & $2.97 \pm 0.14$ & $2.19 \pm 0.86$ & $2.50 \pm 0.76$ & $2.32 \pm 0.93$ \\
\hline High school and above (n:26) & $2.44 \pm 0.95$ & $2.87 \pm 0.72$ & $2.17 \pm 0.74$ & $2.57 \pm 0.59$ & $2.51 \pm 0.87$ & $2.64 \pm 0.69$ & $2.69 \pm 0.59$ & $2.67 \pm 0.34$ & $2.98 \pm 0.45$ & $3.06 \pm 0.23$ & $2.85 \pm 0.71$ & $3.12 \pm 0.54$ & $2.30 \pm 0.77$ \\
\hline$p$ value & 0.474 & 0.294 & 0.309 & 0.116 & 0.024 & 0.084 & 0.769 & 0.264 & 0.666 & 0.130 & 0.023 & 0.030 & 0.607 \\
\hline \multicolumn{14}{|l|}{ Economic condition** } \\
\hline Income higher than expense (n:11) & $2.68 \pm 1.18$ & $2.81 \pm 0.76$ & $1.98 \pm 0.64$ & $2.28 \pm 0.65$ & $2.53 \pm 0.72$ & $2.76 \pm 052$ & $2.60 \pm 063$ & $2.54 \pm 0.47$ & $2.82 \pm 0.43$ & $2.96 \pm 0.15$ & $2.68 \pm 0.82$ & $3.00 \pm 0.71$ & $2.20 \pm 0.91$ \\
\hline Income equal to expense $(n: 26)$ & $2.12 \pm 0.76$ & $2.81 \pm 0.79$ & $2.15 \pm 0.64$ & $2.60 \pm 0.57$ & $2.22 \pm 0.90$ & $2.53 \pm 0.79$ & $2.77 \pm 0.47$ & $2.64 \pm 0.33$ & $3.01 \pm 0.44$ & $3.08 \pm 0.22$ & $2.21 \pm 0.69$ & $2.59 \pm 0.67$ & $2.33 \pm 0.83$ \\
\hline Income lower than expense (n:8) & $2.44 \pm 0.98$ & $2.60 \pm 0.67$ & $2.06 \pm 0.74$ & $2.40 \pm 0.65$ & $2.06 \pm 1.21$ & $2.29 \pm 0.75$ & $2.75 \pm 0.58$ & $2.58 \pm 0.42$ & $3.12 \pm 0.27$ & $3.00 \pm 0.20$ & $2.74 \pm 0.98$ & $2.91 \pm 0.79$ & $2.40 \pm 1.03$ \\
\hline$p$ value & 0.447 & 0.737 & 0.876 & 0.334 & 0.508 & 0.275 & 0.929 & 0.794 & 0.199 & 0.437 & 0.249 & 0.385 & 0.853 \\
\hline \multicolumn{14}{|l|}{ Number of children* } \\
\hline $1(n: 17)$ & $2.44 \pm 0.96$ & $2.90 \pm 0.85$ & $2.14 \pm 0.70$ & $2.50 \pm 0.65$ & $2.55 \pm 0.83$ & $2.56 \pm 0.75$ & $2.75 \pm 0.54$ & $2.72 \pm 0.36$ & $2.89 \pm 0.49$ & $3.07 \pm 0.23$ & & & \\
\hline 2 and more (n:28) & $2.28 \pm 0.93$ & $2.66 \pm 0.67$ & $2.05 \pm 0.64$ & $2.45 \pm 0.60$ & $2.05 \pm 1.00$ & $2.48 \pm 0.73$ & $2.71 \pm 0.54$ & $2.53 \pm 0.38$ & $3.07 \pm 0.33$ & $3.00 \pm 0.19$ & & & \\
\hline$p$ value & 0.548 & 0.459 & 0.933 & 0.733 & 0.090 & 0.546 & 0.783 & 0.213 & 0.113 & 0.449 & & & \\
\hline
\end{tabular}

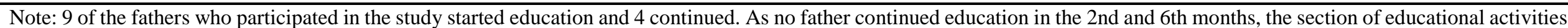
was not analyzed.

*Mann Whitney U Test **Kruskal Wallis Test 
Table 5a. The effect of some socio-demographic variables on the functional status of mothers

\begin{tabular}{|c|c|c|c|c|c|c|c|c|c|}
\hline \multirow{2}{*}{ Variables } & \multicolumn{2}{|c|}{ Household responsibilities } & \multicolumn{2}{|c|}{ Social and community activities } & \multicolumn{2}{|c|}{ Infant care responsibilities } & \multicolumn{2}{|c|}{ Personal care activities } & \multirow{2}{*}{$\begin{array}{l}\text { Occupational activities } \\
\text { 6th month }\end{array}$} \\
\hline & 2nd month & 6th month & 2nd month & 6th month & 2nd month & 6th month & 2nd month & 6th month & \\
\hline 29 year and younger (n:9) & $2.55 \pm 0.57$ & $3.00 \pm 0.35$ & $1.86 \pm 0.44$ & $2.06 \pm 0.47$ & $3.74 \pm 0.28$ & $3.87 \pm 0.18$ & $2.71 \pm 0.34$ & $3.11 \pm 0.32$ & $2.80 \pm 0.60$ \\
\hline 30 year and over (n:36) & $2.62 \pm 0.42$ & $3.03 \pm 0.52$ & $1.76 \pm 0.35$ & $2.19 \pm 0.54$ & $3.76 \pm 0.25$ & $3.80 \pm 0.28$ & $2.69 \pm 0.50$ & $3.06 \pm 0.25$ & $2.88 \pm 0.39$ \\
\hline$p$ value & 0.882 & 0.954 & 0.514 & 0.674 & 0.916 & 0.642 & 0.991 & 0.720 & 0.833 \\
\hline \multicolumn{10}{|l|}{ Education* } \\
\hline$\overline{\text { Secondary school and below (n:17) }}$ & $2.70 \pm 0.54$ & $2.84 \pm 0.44$ & $1.77 \pm 0.33$ & $2.00 \pm 0.32$ & $3.71 \pm 0.26$ & $3.81 \pm 0.28$ & $2.71 \pm 0.42$ & $3.11 \pm 0.29$ & - \\
\hline High school and above (n:28) & $2.52 \pm 0.46$ & $3.12 \pm 0.42$ & $1.83 \pm 0.43$ & $2.21 \pm 0.59$ & $3.77 \pm 0.26$ & $3.85 \pm 0.22$ & $2.70 \pm 0.44$ & $3.06 \pm 0.78$ & $2.86 \pm 0.45$ \\
\hline$p$ value & 0.385 & 0.043 & 0.728 & 0.166 & 0.339 & 0.737 & 0.787 & 0.739 & 0.859 \\
\hline \multicolumn{10}{|l|}{ Economic condition $* *$} \\
\hline Income higher than expense (n:11) & $2.57 \pm 0.44$ & $3.14 \pm 0.47$ & $1.89 \pm 0.51$ & $2.14 \pm 0.59$ & $3.66 \pm 0.35$ & $3.77 \pm 0.27$ & $2.71 \pm 0.44$ & $3.07 \pm 0.30$ & $2.84 \pm 0.45$ \\
\hline Income equal to expense (n:26) & $2.54 \pm 0.45$ & $3.07 \pm 0.40$ & $1.81 \pm 0.37$ & $2.14 \pm 0.55$ & $3.78 \pm 0.24$ & $3.85 \pm 0.19$ & $2.75 \pm 0.39$ & $3.08 \pm 0.30$ & $2.88 \pm 0.46$ \\
\hline Income lower than expense (n:8) & $2.49 \pm 0.68$ & $2.66 \pm 0.39$ & $1.70 \pm 0.28$ & $2.07 \pm 0.18$ & $3.79 \pm 0.14$ & $3.85 \pm 0.35$ & $2.53 \pm 054$ & $3.10 \pm 0.21$ & \\
\hline$p$ value & 0.615 & 0.051 & 0.685 & 0.919 & 0.626 & 0.408 & 0.542 & 0.988 & 0.915 \\
\hline \multicolumn{10}{|l|}{ Number of children* } \\
\hline$\overline{1(\mathrm{n}: 17)}$ & $2.50 \pm 0.47$ & $3.11 \pm 0.36$ & $1.78 \pm 0.46$ & $2.10 \pm 0.45$ & $3.71 \pm 0.28$ & $3.87 \pm 0.18$ & $2.61 \pm 0.42$ & $3.03 \pm 0.29$ & $2.85 \pm 0.64$ \\
\hline 2 and more (n:28) & $2.65 \pm 0.50$ & $2.96 \pm 0.48$ & $1.82 \pm 0.35$ & $2.15 \pm 0.55$ & $3.77 \pm 0.25$ & $3.81 \pm 0.27$ & $2.76 \pm 0.43$ & $3.11 \pm 0.28$ & $2.86 \pm 0.30$ \\
\hline p value & 0.425 & 0.230 & 0.517 & 0.980 & 0.303 & 0.796 & 0.188 & 0.418 & 0.913 \\
\hline
\end{tabular}

*Mann Whitney U Test **Kruskal Wallis Test

Note: As no woman started working in the 2nd month, the section of occupational activities was not analyzed. 
Table 5b. The effect of some socio-demographic variables on the functional status of mothers

\begin{tabular}{|c|c|c|c|c|c|c|c|c|c|}
\hline \multirow[t]{2}{*}{ Variables } & \multicolumn{2}{|c|}{ Household responsibilities } & \multicolumn{2}{|c|}{ Social and community activities } & \multicolumn{2}{|c|}{ Infant care responsibilities } & \multicolumn{2}{|c|}{ Personal care activities } & \multirow{2}{*}{$\begin{array}{c}\text { Occupational activities } \\
6 . \text { month }\end{array}$} \\
\hline & 2. month & 6. month & 2. month & 6. month & 2. month & 6. month & 2. month & 6. month & \\
\hline Delivery method & & & & & & & & & \\
\hline $\begin{array}{l}\text { Normal birth (n:11) } \\
\text { Caesarean -section (n:34) }\end{array}$ & $\begin{array}{l}2.75 \pm 0.57 \\
2.54 \pm 0.46\end{array}$ & $\begin{array}{l}2.79 \pm 0.27 \\
3.09 \pm 0.47\end{array}$ & $\begin{array}{l}1.89 \pm 0.36 \\
1.78 \pm 0.41\end{array}$ & $\begin{array}{l}1.96 \pm 0.41 \\
2.18 \pm 0.53\end{array}$ & $\begin{array}{l}3.72 \pm 0.29 \\
3.76 \pm 0.25\end{array}$ & $\begin{array}{l}3.80 \pm 0.24 \\
3.84 \pm 0.24\end{array}$ & $\begin{array}{l}2.81 \pm 0.33 \\
2.66 \pm 0.46\end{array}$ & $\begin{array}{l}3.05 \pm 0.23 \\
3.09 \pm 0.30\end{array}$ & $\begin{array}{c}- \\
286+0,43\end{array}$ \\
\hline$p$ value & 0.153 & 0.042 & 0.372 & 0.350 & 0.712 & 0.569 & 0.307 & 0.629 & - \\
\hline \multicolumn{10}{|l|}{ State of intending pregnancy } \\
\hline $\begin{array}{l}\text { Intended and planned (n:36) } \\
\text { Intended but unplanned (n:9) }\end{array}$ & $\begin{array}{l}2.63 \pm 0.47 \\
2.41 \pm 0.58\end{array}$ & $\begin{array}{l}3.03 \pm 0.43 \\
2.98 \pm 0.51\end{array}$ & $\begin{array}{l}1.83 \pm 0.39 \\
1.71 \pm 0.40\end{array}$ & $\begin{array}{l}2.16 \pm 0.55 \\
2.02 \pm 0.30\end{array}$ & $\begin{array}{l}3.75 \pm 0.27 \\
0.75 \pm 0.22\end{array}$ & $\begin{array}{l}3.82 \pm 0.22 \\
3.87 \pm 0.33\end{array}$ & $\begin{array}{l}2.72 \pm 0.41 \\
2.61 \pm 0.52\end{array}$ & $\begin{array}{l}3.09 \pm 0.31 \\
3.04 \pm 0.13\end{array}$ & $\begin{array}{l}2.86 \pm 0.45 \\
2.80 \pm 0.40\end{array}$ \\
\hline$p$ value & 0.159 & 0.932 & 0.416 & 0.559 & 0.837 & 0.193 & 0.679 & 0.471 & 0.859 \\
\hline \multicolumn{10}{|c|}{ Contribution to infant care from the outside } \\
\hline $\begin{array}{l}\text { Yes }(\mathrm{n}: 25) \\
\text { No }(\mathrm{n}: 20)\end{array}$ & $\begin{array}{l}2.50 \pm 0.47 \\
2.71 \pm 0.50\end{array}$ & $\begin{array}{l}3.08 \pm 0.47 \\
2.94 \pm 0.40\end{array}$ & $\begin{array}{l}1.79 \pm 0.42 \\
1.84 \pm 0.37\end{array}$ & $\begin{array}{l}2.24 \pm 0.60 \\
2.00 \pm 0.34\end{array}$ & $\begin{array}{l}3.76 \pm 0.26 \\
3.75 \pm 0.27\end{array}$ & $\begin{array}{l}3.84 \pm 0.24 \\
3.83 \pm 0.25\end{array}$ & $\begin{array}{l}2.69 \pm 0.35 \\
2.72 \pm 0.52\end{array}$ & $\begin{array}{l}3.10 \pm 0.31 \\
3.06 \pm 0.26\end{array}$ & $\begin{array}{l}2.87 \pm 0.36 \\
2.80 \pm 0.84\end{array}$ \\
\hline$p$ value & 0.192 & 0.301 & 0.543 & 0.247 & 0.962 & 0.890 & 0.705 & 0.676 & 0.894 \\
\hline \multicolumn{10}{|c|}{ Contribution to household chores from the outside } \\
\hline $\begin{array}{l}\text { Yes }(\mathrm{n}: 24) \\
\text { No }(\mathrm{n}: 21)\end{array}$ & $\begin{array}{l}2.48 \pm 0.53 \\
2.71 \pm 0.42\end{array}$ & $\begin{array}{l}3.05 \pm 0.45 \\
2.98 \pm 0.44\end{array}$ & $\begin{array}{l}1.87 \pm 0.40 \\
1.74 \pm 0.39\end{array}$ & $\begin{array}{l}2.12 \pm 0.52 \\
2.14 \pm 0.51\end{array}$ & $\begin{array}{l}3.74 \pm 0.27 \\
3.76 \pm 0.25\end{array}$ & $\begin{array}{l}3.77 \pm 0.29 \\
3.91 \pm 0.13\end{array}$ & $\begin{array}{l}2.62 \pm 0.36 \\
2.79 \pm 0.49\end{array}$ & $\begin{array}{l}3.10 \pm 0.27 \\
3.05 \pm 0.30\end{array}$ & $\begin{array}{l}2.96 \pm 0.36 \\
2.70 \pm 0.52\end{array}$ \\
\hline p value & 0.218 & 0.522 & 0.333 & 0.885 & 0.698 & 0.105 & 0.160 & 0.398 & 0.383 \\
\hline
\end{tabular}

Mann Whitney U Test $\quad * *$ Kruskal Wallis Test

Note: As no woman started working in the 2nd month, the section of occupational activities was not analyzed. 


\section{DISCUSSION, CONCLUSION AND SUGGESTIONS}

Preparing both women and their partners for the challenges of parenthood before, during and after the birth is very important in terms of maternal, child, and family health. The preparation of fathers to fatherhood, as well as mothers for motherhood, positively affects family health (Apay Ejder \& Pasinlioğlu, 2009; Beji et al., 2003; Özkan \& Sevil, 2007; Sevil \& Özkan, 2010).

According to the results of this study, positive changes developed in the functional status of women and their partners within the months marking the postpartum period. Women were found to have higher mean scores in the areas of household activities, social and community activities and self-care activities in the postpartum sixth month, compared to those of the postpartum eighth week. However, there was no significant difference between the postpartum second and sixth months in terms of infant care activities and occupational activities. According to some studies in the academic literature, the postpartum functional status of women shows significant development in the months following the birth (McVeigh, 1997; McVeigh \& Chaboyer, 2002; Özkan \& Sevil, 2007; Şanlı \& Öncel, 2014; Tulman et al., 1990). While postpartum physical recovery generally takes six weeks to complete, adapting to the increasing responsibilities related the postpartum period, like parenthood and infant care, tends to require a longer time (Beji et al., 2003). Apay and Pasinlioğlu (2009) reported in their study that after childbirth, the scores obtained on the subscale of social and community activities increased. In the study conducted by Şanlı and Öncel (2014) they found that women become capable of fulfilling their responsibilities, primarily those related to infant care, followed by housework, within the first sixth months following childbirth.

In this study, a significant increase was observed in the household activities, social and community activities and child care activities of fathers in the postpartum sixth month, as compared to the postpartum eighth week. On the other hand, no significant difference was discovered between the months in terms of the mean scores obtained in the areas of infant care activities, self-care activities, occupational activities, and educational activities. In one study on the functional status of fathers, it was noted that following childbirth, for the most part, a majority of fathers maintained their regular participation level in household and intrafamilial activities, while for some, there was a very slight increase in their responsibility (McVeigh, 2001). Other studies have reported that husband's whose wives supported their involvement in infant care had more infant care responsibilities. An examination of the related expectations of couples within the first three weeks after the birth revealed that fathers felt unsupported in infant care when it came to actions (St John, Cameron, \& McVeigh, 2005; Sevil \& Özkan, 2010). There are only a very limited number of studies in Turkey regarding the functional status of fathers. In one of them, Sevil and Özkan (2010) found there to be no difference in the functional status of fathers at the time their wives were pregnant and during the postpartum period. In Turkey, men are not expected to participate in trainings with their wives before and after the birth, and there are only limited opportunities available for men to prepare them for the role of fatherhood. These drawbacks are believed to be related to the assumption that there is no difference between the functional status of "expectant" fathers and new fathers.

The present study found that age had no impact on the functional status of women and their partners. In contrast, Apay and Pasinlioglu (2009) in their study, determined that with the increase of age, functional status scores decreased, as individuals avoided doing certain activities or did a lower rate of activities, possibly as a result of age-related physical inability and/or health disorders. In the study by Sevil and Özkan (2010), it was reported that compared to younger age groups, elderly fathers were better at occupational activities. The same study also found there to be a negative correlation between the age of fathers and social-community and self-care activities, and a positive relation between the age of fathers and household 
activities. Şanlı and Öncel (2014) discovered in their study that as women aged their functional status increased, helping them to feel more responsible and experienced regarding infant care and household activities, particularly in cases where the women had a higher number of children. This last finding, as it relates to its positive effect on functional status, can be attributed to the fact that the women's exposure to postpartum functions helped them to be better prepared to handle the process. The women and their partners who participated in the present study were in the fertile age group, which could serve to explain the insignificant impact of age on functional status.

The results from this study found that educational level had a positive effect on functional status. As the educational level increased, the mean scores obtained by the women in the area of household activities were higher in the postpartum sixth month. Fathers with a higher educational level were determined to have higher mean scores in the area of infant care activities in the postpartum eighth week, and in the area of child care activities in both the postpartum second and the postpartum sixth month. Contrary to the results of the present study, Tulman et al., (1990) reported that women with a higher educational level had lower functional status than that of women with a lower educational level. Posmontier (2008) stated in his study that as the educational level increased, the self-care of women increased. Furthermore, in a study by Şanlı and Öncel (2014), primary school graduate women were observed to have a higher functional status in the area of infant care responsibilities in the postpartum sixth week, while university and higher education graduate women had a higher functional status in the area of self-care activities in the postpartum third and the postpartum sixth months when compared to other groups. In examining the studies on the functional status of fathers, McVeigh (2001) found that there was an increase in the functional status of fathers who had a higher educational level. Similarly, Sevil and Özkan (2010) also determined that the functional status of fathers was positively affected by a higher educational level. Although other studies have shown different results, the present study observed that women who had a caesarean section had stable mean scores in the area of household activities in the postpartum eighth week but higher mean scores in the same area in the postpartum sixth month, compared to those who gave normal birth. However, it was observed that the delivery method had no effect on the other activities.

Tulman and Fawcet (1988), in their study, determined that functions were regained after the postpartum sixth week, and that in general, women who gave vaginal birth were able to recover faster than those who had a cesarean section. In a related study, Beji et al., (2003) found that women who gave vaginal birth took on the infant responsibility earlier. Lastly, Şanlı and Öncel (2014) determined that women who gave vaginal birth regained their functional status of infant care faster than those who had a caesarean section. In the present study, the higher scores obtained by women in the area of household activities were associated with the higher level of support received regarding housework in the postpartum period.

The present study, found that the number of children, planned or unplanned status of pregnancy, and the provision of outside support for household activities and infant care did not affect the functional status. Tulman et al., (1990) in their study, observed that the increased number of pregnancies and births resulted in increased social-community activities and selfcare activities. The study by Şanlı and Öncel (2014) determined that the number of children affected the postpartum functional status of women in the postpartum sixth week and the postpartum third month. Moreover, the same study found that with the increased number of children, household activities, infant care and total functional status increased, while the functional status regarding self-care decreased, with the difference being maintained in the postpartum sixth month. To continue, in the studies by Apay and Pasinlioğlu (2009) and Özkan and Sevil (2007), the women who had planned pregnancies were shown to have higher 
postpartum functional status. On the other hand, Şanl1 and Öncel (2014) found that the women who had unplanned pregnancies had higher mean scores in the area of infant care in the postpartum sixth week, in the area of household activities in the postpartum third month, and in the area of self-care activities in the postpartum third and sixth months. In the present study, the variable of planned/not planned pregnancy was shown to not affect the functional status of women in the postpartum second and sixth month, a finding associated with the fact that all the women who participated in the study had planned pregnancies.

Women who received support in their household activities have been shown to have less domestic activities, lower total postpartum functional status and less infant care responsibilities (Apay et al., 2009; Özkan et al., 2007). In the study conducted by Mc Veigh (2000b), it was determined that women who received social support in their postpartum sixth week, third month, and sixth month had significantly higher household, social-community and self-care activities. Özkan and Sevil (2007), in their study, found that women who received support in infant care had higher functional status regarding infant care. In the study conducted by Apay and Pasinlioğlu (2009), it was observed that women who received support in infant care had lower functional status regarding household activities and infant care. Further, Şanlı and Öncel (2014), determined that women who received no support in infant care and household activities returned to their functional status earlier. In the same study, it was reported that the lower functional status of women who received support in infant care and household activities was associated with the fact that routine work that had at once been done by them was now undertaken by supporters. In contrast to the studies cited above, the present study found there to be no difference in the women' functional status in terms of having/not having support in household activities and infant care. Considering the results of this study and the other studies mentioned above, it was concluded that having/not having outside help in household activities and infant care produces different effects on the women' functional status.

Nurses and midwives have important functions in the transition process of parents to their changing roles and in the development of healthy children. In Turkey, by identifying the functional status of women and their partners after the birth and by determining the factors affecting the functional status, women and their partners will have the opportunity to be given full support and thereby be better adapted to the postpartum period. With that said, it is important that nurses and midwives support parents throughout the pregnancy process, including up to the completion of the transition to their new roles of mother and father, and help them to make the changes necessary to prepare them for this new period of their life.

\section{ORCID}

Sevgi Özkan (D) https://orcid.org/0000-0001-8385-210X

Sinem Göral Türkcü (D) https://orcid.org/0000-0003-1574-0186

Pınar Serçekuş Ak (D) https://orcid.org/0000-00029326-3453

\section{REFERENCES}

Anderson, A.M. (1996). The father- infant releationship; becoming connected. Journal of the Society of Pediatric Nurses, 1(2), 83-92.

Apay Ejder, S., \& Pasinlioğlu, T. A. (2009). Sectional study: the invertigation of functional situations of the women after the labor. Hemşirelikte Araştırma Geliştirme Dergisi, 1(1), 20-29.

Barclay, L., Donovan, J., \& Genovese, A. (1996). Men's experiences during their partner's first pregnancy: a grounded theory analysis. Australian Journal of Advanced Nursing, 13(3), 12-24. 
Barclay, L., \& Lupton, D. (1999). The experiences of new fatherhood: a socio-cultural analysis. Journal of Advanced Nursing, 29(4), 1013-1020.

Başbakkal, Z. (1999). Babaların bebek bakımındaki rolü (Doktora Tezi). [Role of parents in baby care] (Doctoral Thesis). Ege Üniversitesi, Institute of Health Sciences, Izmir.

Beji, N.K., Coşkun, A., \& Yıldırım, G. (2003). Effects of childbirth on the functional ability of women. Hemşirelikte Araştırma Geliştirme Dergisi, 1, 22-29.

Fawcett, J., \& Tulman, L.(1990). Building a programme of research from the roy adaptation model of nursing, Journal of Advenced Nursing, 15(6), 720-725.

Fawcett, J., Tulman, L., \& Myers, S. (1988). Development of the inventory of functional status after childbirth. Journal of Nurse-Midwifery, 33(6), 252-260.

Fichardt, A.E., Van Wyk, N.C., \& Weich, M. (1994). The needs of postpartum women. Curationis, 17(1), 15-21.

Gjerdingen, D.K., \& Chaloner, K.(1994). Mothers' experience with houshold roles and social support during the first postpartum years. Women Health, 21(4), 54-74.

Henderson, A.D, \& Brouse, A.J. (1991). The experiences of new fathers during the first 3 weeks of life. Journal of Advanced Nursing,16(3), 293-298.

Herbert, P. (1994). Support of first- time mothers in three months after birth. Nursing Times, 90(24), 36-37.

Hodnett, E.D. (1996). Nursing support of the laboring woman. Journal of Obstetric, Gynecologic and Neonatal Nursing, 25(3), 257-264.

McVeigh, C.A. (1997). Functional status after childbirth: a comparison of Australian women from english and non-english speaking backraunds. Australian College of Midwives Incorporated Journal, 10(2), 15-21.

McVeigh, C. A. (2000a). Anxiety and functional status after childbirth. Australian College of Midwives Journal, 13(1), 14-18.

McVeigh, C.A. (2000b). Satisfaction with social support and functional status after childbirth. Maternal Child Nursing Journal, 25(1), 25-30.

McVeigh, C.A. (2001). Functional status after fatherhood, an Australian study. Journal of Obstetric, Gynecologic and Neonatal Nursing, 31, 32-38.

McVeigh, C.A., Chaboyer, W. (2002). Reability and validity of inventory of functional status after childbirth when used in Australian population. Nursing \& Health Sciences, 4(3), 107-112.

Özkan, S., \& Sevil, Ü. (2007). The study of validity and reliability of inventory of functional status after childbirth. TSK Korиyиси Hekimlik Bülteni, 6, 199-208.

Posmontier, B. (2008). Functional status outcomes in mothers with and without postpartum Depression. Journal of Midwifery \& Women's Health, 53(4), 310-318.

Sevil, Ü., \& Özkan, S. (2010). Psychometric properties of a Turkish version of the inventory of functional status-fathers (IFS-F). Turkiye Klinikleri Journal of Nursing Science, 2, 16-23.

St John, W., Cameron, C, \& McVeigh. C. (2005). Meeting the challenge of new fatherhood during the early weeks. Journal of Obstetric, Gynecologic \& Neonatal Nursing, 34(2), 180-189.

Şanlı,Y., \& Öncel, S. (2014). Kadınların doğum sonrası fonksiyonel durumları ve etkileyen faktörlerin belirlenmesi [Evaluation of the functional status of woman after childbirth and effective factors]. Journal of Turkish Obstetric and Gynecology Society, 2, 105-114.

Tulman, L., \& Fawcett, J. (1988). Return of functional ability after childbirth. Nursing Research, 37, 70-75. 
Tulman, L., Fawcett, J., Groblewski, L., \& Silverman, L. (1990). Changes in functional status after childbirth. Nursing Research, 39(2), 70-75.

Tulman, L.J., Fawcett, J., \& Weiss, M. (1993). The inventory of functional status-fathers: development and psychometric testing. Journal of Nurse-Midwifery, 38, $276-282$. 